\title{
Stress assessment based on EEG univariate features and functional connectivity measures
}

\author{
J F Alonso ${ }^{1,2,3}$, S Romero ${ }^{1,3}$, M R Ballester ${ }^{4,5,6}$, R M Antonijoan ${ }^{4,5,6,7}$, and M A Mañanas ${ }^{1,2,3}$ \\ 1 Biomedical Engineering Research Centre (CREB), Department of Automatic Control (ESAII), \\ Universitat Politècnica de Catalunya (UPC), Barcelona, Spain. \\ 2 Barcelona College of Industrial Engineering (EUETIB), UPC, Barcelona, Spain. \\ 3 Biomedical Research Networking Center in Bioengineering, Biomaterials and Nanomedicine \\ (CIBER-BBN), Spain. \\ 4 Drug Research Center (CIM), Institute of Biomedical Research (IIB), Research Institute (RI), \\ Barcelona, Spain. \\ 5 Hospital de la Santa Creu i Sant Pau, Barcelona, Spain. \\ 6 Department of Pharmacology and Therapeutics, Universitat Autònoma de Barcelona (UAB), \\ Barcelona, Spain. \\ 7 Biomedical Research Networking Center in Mental Health (CIBERSAM), Spain.
}

E-mail: joan.francesc.alonso@upc.edu

\begin{abstract}
The biological response to stress originates in the brain but involves different biochemical and physiological effects. Many common clinical methods to assess stress are based on the presence of specific hormones and on features extracted from different signals, including electrocardiogram, blood pressure, skin temperature, or galvanic skin response. The aim of this paper was to assess stress using EEG-based variables obtained from univariate analysis and functional connectivity evaluation. Two different stressors, Stroop test and sleep deprivation, were applied to 30 volunteers to find common EEG patterns related to stress effects. Results showed a decrease of high alpha power (11 to $12 \mathrm{~Hz}$ ), an increase in the high beta band ( 23 to $36 \mathrm{~Hz}$, considered a busy brain indicator), and a decrease in the approximate entropy. Moreover, connectivity showed that the high beta coherence and the interhemispheric nonlinear couplings, measured by the cross mutual information function, increased significantly for both stressors, suggesting that useful stress indexes may be obtained from EEG-based features.
\end{abstract}

Keywords. Stress; EEG; Stroop test; sleep deprivation; spectral analysis; mutual information function. 


\section{Introduction}

Stress is part of life. Life itself offers specific challenges for our minds, bodies and emotions. Responses to stress are observed in multiple psychophysiological systems, such as the cerebral, endocrine, and immune systems (Woolfolk et al. 2007). Once the brain detects an emotional, mental or physical tension, a stress response based on biochemical reactions with different effects is triggered: several hormones and antibodies are secreted, such as adrenaline, immunoglobulin A (IgA), and cortisol; and heart rate and blood pressure increase (Seo and Lee 2010). This response is adequate in the short-term but a long-lasting stressing situation can have detrimental effects on health, because the stress-related corticosteroid hormones can weaken the effectiveness of the immune system and make people more susceptible to infection. Long-term stress can also increase the risk of illnesses such as depression, heart disease or diabetes (Pruett 2003). Unfortunately, our current high-speed daily life causes the brain to be in a constant state of physiological arousal over perceived threat and therefore stress responses are frequently activated, leading to chronic stress states (Lederbogen et al. 2011).

Since the origin of stress is in the brain, its activity acquired by the electroencephalogram (EEG) becomes an interesting tool to detect and analyse human stress (Seo and Lee 2010). Past studies have investigated the changes in EEG signal under a single stressing condition. Researchers have focused on the search of changes in spectral variables because concentration and mental load are principally characterized by an increase of beta activity. Thus, the EEG under these stressors reflects a decrease of alpha power in the range from 11 to $12 \mathrm{~Hz}$ and an increase of beta activity, especially in the $23-36 \mathrm{~Hz}$ range which is considered a 'busy brain' indicator (Tran et al. 2007). Other works have used regularity indexes such as entropies to assess changes in the EEG time series under stress, evidencing regularity increases under fatigue and stress, and decreases under alertness (Tran et al. 2007).

In addition to these results, there is abundant evidence supporting the emotion model concerning hemispheric specialization: while left hemisphere is more involved in processing positive emotions, the right hemisphere is considered to handle negative stimuli (Davidson 1993). The prefrontal cortex plays an important role in the emotional and motivational processes (Davidson 2004). According to this model, asymmetry in frontal alpha activity is considered a reflection of emotions, and thus constitutes a measure associated with feelings. In fact, statistical differences of the frontal EEG alpha asymmetry have been observed under depression (Hinrikus et al. 2009), body massage (Chang et al. 2012), examination stress (Lewis et al. 2007), and sleep deprivation (Ferreira et al. 2006).

Hence, this asymmetric emotion model is related to the relationship between neurophysiological events recorded at different locations on the scalp, or, in other words, functional connectivity of the brain. Few studies have dealt with the assessment of connectivity using EEG during stress tests, and these works have mainly focused on the evaluation of single stress-related conditions, such as sleep deprivation and fatigue, whose effects on EEG connectivity have been revealed by means of linear techniques, such as coherence (Hinrikus et al. 2009, Papadelis et al. 2007), directed transfer function (De Gennaro et al. 2005), or more sophisticated nonlinear methods such as synchronization likelihood (Kar et al. 2011), phase-lag index (Koenis et al. 2011), and averaged cross mutual information function ( $\mathrm{Na}$ et al. 2006). These studies have reported, among other results, decreases of entropies and mutual information in few pairs of electrodes, and have shown frequency-specific changes in the functional network topology of the brain. However, it is difficult to interpret which of these changes were exclusively related to the stressor or were also caused by the fatigue associated to sleep deprivation.

The main objective of the present work was to study changes in cerebral activity and connectivity on the whole scalp EEG during two different stress-related experiments. In this work, entropy, frontal asymmetry, and spectral measures were calculated, whereas connectivity assessment was carried out through linear and nonlinear measures based on cross mutual information function (Alonso et al. 2010). The stress conditions, namely the Stroop test (Manuck et al. 1991, Tulen et al. 1989) and sleep deprivation (Irwin et al. 1996), are considered a psychological stressor and a physical stressor associated with fatigue, respectively, and were applied to investigate abnormalities and common EEG patterns which could be characteristic of stress. 


\section{Materials and methods}

\subsection{Subjects and instrumentation}

Thirty-two young healthy volunteers were included in the study. Volunteers were reported to be in good health confirmed by medical history and examination, laboratory tests, ECG and urinalysis, and without psychiatric or neurological disorders. Subjects who had trouble sleeping (assessed by polysomnography), or extreme punctuations of anxiety (assessed by the STAI-trait and STAI-state tests), or high vulnerability to stress (assessed by the perceived stress questionnaire, PSQ, and the stress reactivity index, IRE-32), as well as those with recent surgery, or alcohol or drug abuse, were excluded from the study. Finally, only 30 subjects aged between 18 and 35 years (mean 26.53 years) were able to complete the study. They did not take any medication in the two weeks prior to the study nor until the end of the experimental sessions, and abstained from alcohol, tobacco, and caffeinated drinks for $48 \mathrm{~h}$ before each experimental day.

EEG recordings were acquired from a scalp cap using 19 electrodes according to the international 10/20 system on the following locations: Fp1, Fp2, F7, F3, Fz, F4, F8, T3, C3, Cz, C4, T4, T5, P3, Pz, $\mathrm{P} 4, \mathrm{~T} 6, \mathrm{O} 1$ and $\mathrm{O} 2$, referenced to averaged mastoids. Additionally, vertical and horizontal EOG (VEOG and HEOG, respectively) signals were recorded as auxiliary signals for ocular artefact reduction. The VEOG was obtained from mid-forehead $(2.5 \mathrm{~cm}$ above the interpupillary line) and from the average of one electrode below the left eye and another below the right eye $(2.5 \mathrm{~cm}$ below the interpupillary line), whereas the HEOG signal was acquired from the outer canthi. The EEG and EOG signals were analogically band-pass filtered between 0.1 and $70 \mathrm{~Hz}$ and recorded with a sampling frequency of $250 \mathrm{~Hz}$, by means of a BrainAmp amplifier. All signals were digitally filtered between 0.5 and $35 \mathrm{~Hz}$ and resampled to $100 \mathrm{~Hz}$ for subsequent analysis.

\subsection{Experimental stress tests}

The study was approved by the Ethics Committee of the Hospital de la Santa Creu i Sant Pau and the Spanish Ministry of Health, and was conducted following the principles stated in the Declaration of Helsinki and the guidelines of good clinical practice. All volunteers gave their written informed consent to participate. Figure 1 shows a schematic diagram of the experimental trial. Volunteers arrived at $7 \mathrm{am}$ to the laboratory and remained all day under medical supervision so that their diet and activity were controlled. Volunteers were kept awake for $30 \mathrm{~h}$. A 10 min Stroop test was performed on the first day at the arrival to the lab. Three-minute EEG recordings with eyes closed were acquired before (Re1) and after (Re2) performing the Stroop test, and after the 30-hour sleep deprivation (Re3). Special care was taken to guarantee high quality EEG signals throughout the duration of the study by ensuring that the skullcap was placed correctly and electrode impedances were kept below $5 \mathrm{k} \Omega$ before each recording.

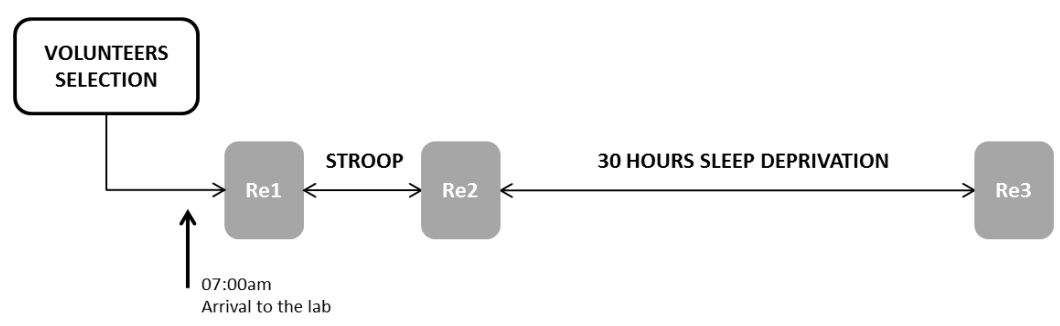

Figure 1. Schematic diagram of the planning of the experimental study indicating the time points where EEG recordings were acquired. Baseline register is referred as Re1, post-Stroop register is Re2, and post-deprivation recording is Re3.

The Stroop test is an outcome of our flexibility of attention and its effects are related to the ability of most people to read words more quickly and automatically than they can name colours (Stroop, 1935). If a word is displayed in a colour different from the colour the word actually refers, it is more difficult to voice the colour, and even when asked to name it we tend to say the name the word represents. The task of making an appropriate response and its related cognitive mechanism allow us to focus our thoughts, but when placed under continual demand this ability tires, reducing our mental 
effectiveness and creating stress. Hence, the Stroop task causes irritability and impulsivity that result in regrettable behaviour, impatience that has us making poor decisions, and distractibility that allows the immediate environment to have a greatly magnified influence on our decisions. The Stroop test has already been used as a laboratory stressor and typically results in increased cardiac activity (heart rate and blood pressure), skeletal muscle vasodilation, and altered immune response (Hamer et al. 2003). For example, after 20 minutes of Stroop test a reduction of mitogenesis and alterations in various circulating lymphocyte populations (CD4, CD8 and CD19) has been observed (Manuck et al. 1991).

On the other hand, sleep is a natural neural state of rest occurring at regular intervals and is necessary for the maintenance of health. Relatively brief sleep deprivation can be considered a stressor that has consequences for the brain, as well as many other systems in the human body (McEwen et al. 2006). It is known that sleep deprivation can cause fatigue, irritability, confusion and other psychological effects (Taheri et al. 2004). Moreover, there is increasing evidence not only for cognitive impairment resulting from sleep deprivation, but also for the immune system showing altered levels of cytokines, lymphocytes and oxidative stress markers. Some studies have presented changes in the pattern of white blood cells as a result of sleep deprivation, as an indicator of the stress response of the body (Bollinger et al. 2009, Ackermann et al. 2012). For this stressor, blood samples were drawn after EEG recording to perform IgA measurement and lymphocyte cell count.

\subsection{Ocular artefact reduction}

Raw EEG signals were subjected to an ocular artefact reduction process based on blind source separation (BSS). This technique decomposes the multichannel EEG and EOG data into a mixture of source signals. For this purpose, the SOBI (second order blind identification) algorithm was used, which is based on eigenvalue decomposition of time delayed covariance matrices (Belouchrani et al. 1997). After identifying the source signals associated with eye movements, the corrected EEG signals were obtained from the remaining components. The identification of the ocular signal sources was based on frequency and scalp topography analyses as described in previous studies (Romero et al. 2008).

After ocular artefact reduction, all signals were found to be suitable for subsequent analysis and all 3-minute recordings were studied.

\subsection{Univariate analysis}

After computing the BSS-based artefact procedure, spectral analysis was performed for all the EEG channels. Power spectral density (PSD) functions were calculated for the artefact corrected signals by means of a Welch's periodogram using a Hanning window, with an equivalent frequency resolution of $0.1 \mathrm{~Hz}$. PSD curves were quantified into the absolute powers of the common frequency bands: delta ( 0.5 to $3.5 \mathrm{~Hz})$, theta $(3.5$ to $7.5 \mathrm{~Hz})$, alpha $(7.5$ to $13 \mathrm{~Hz})$ and beta $(13$ to $36 \mathrm{~Hz})$. Some additional bands were also obtained according to the relevant literature: alpha1 ( 7.5 to $10.5 \mathrm{~Hz}$ ), high alpha (11 to $12 \mathrm{~Hz}$ ), low beta (13 to $23 \mathrm{~Hz}$ ) and high beta (23 to $36 \mathrm{~Hz}$ ) (Ferreira et al. 2006, Hinrikus et al. 2009, Seo and Lee 2010). Relative power variables were also calculated with respect to the total power $(0.5$ to $36 \mathrm{~Hz}$ ).

The frontal EEG asymmetry index (ASI) was computed by subtracting the natural logarithm of the alpha power of the left hemisphere from that of the right hemisphere:

$$
A S I=\ln (R)-\ln (L)=\ln (R / L)
$$

$R$ and $L$ refer to the right and left corresponding electrodes in the pair of compared electrodes. This approach results in a score which weighs the relative activity of the right and left hemispheres, and whose zero value indicates symmetrical activity.

Thus, a positive value of ASI represents a greater relative right frontal activity associated with an increased trait tendency to respond more intensely to affectively negative stimuli (Coan and Allen 2004).

Moreover, approximate entropy was calculated for all EEG channels in order to assess changes in the irregularity of the signals due to the stress condition. The embedding dimension $\mathrm{m}$ was set to 3 , as recommended for dynamic signals such as EEG, and the radius for nearest neighbour search $\mathrm{r}$ was set to its optimal value, both according to Lu et al. (2008). Approximate entropy is a nonlinear method for 
measuring signal irregularity that has shown its usefulness in different EEG studies (Bruhn et al. 2000, Abásolo et al. 2008).

\subsection{EEG connectivity}

Connectivity measures were also obtained from the corrected signals following artefact reduction. Concretely, magnitude-squared coherence (MSC) and cross mutual information function (CMIF) were computed. MSC, which is a measure of linear correlation as a function of frequency, was calculated according to the following equation:

$$
\operatorname{MSC}(f)=\frac{\left|P_{\xi \eta}(f)\right|^{2}}{P_{\xi \xi}(f) P_{\eta \eta}(f)}
$$

where $P_{\xi \xi}(f)$ and $P_{\eta \eta}(f)$ represent the autospectra of two signals $\xi(t)$ and $\eta(t)$ and $P_{\xi \eta}(f)$ corresponds to their cross-spectrum. The significance level for the MSC was calculated by means of twenty sets of amplitude-adjusted phase-randomized surrogate data (Schreiber and Smith 2000, Alonso et al. 2012). The average value of the MSC function was calculated for the same bands used for spectral variables.

With respect to CMIF, it is considered the nonlinear counterpart of the cross-correlation function, and assesses temporal dependencies between signals in terms of information transfer as a function of a time horizon $(\tau)$. CMIF can be calculated using the following equation to quantify the coupling between two signals $\xi(t)$ and $\eta(t)$ (Alonso et al. 2010):

$$
I_{\xi, \eta}(\tau)=H(\xi(t))+H(\eta(t-\tau))-H(\xi(t), \eta(t-\tau))
$$

where $H(\cdot)$ denotes the Shannon entropy of a signal:

$$
H=-\sum_{i} p_{i} \log _{2}\left(p_{i}\right)
$$

for a discrete probability distribution $\left\{p_{i}\right\}$ of the signal obtained by means of equiquantal partitioning using 8 quantiles. As the CMIF takes into account linear and nonlinear dependence between signals, bivariate amplitude-adjusted phase-randomized surrogate data (Schreiber and Smith 2000) were used to obtain the linear and nonlinear components.

Surrogate data preserve all statistical features of the original data but the property of interest. Bivariate surrogate data were obtained by means of the Fourier Transform (FT) of two signals: 1) preserving the magnitudes of the FT in order to conserve first and second order statistics, that is, their linear properties; 2) synchronously randomizing of the phases of the FT to remove nonlinear properties; and 3) transforming back to the time domain. To avoid spurious nonlinearities, histogram gaussianization and restoration were carried out before and after surrogate data generation (Schreiber and Smith 2000). A set of these surrogates preserves linear stochastic synchronization between signals, and by using the average of 20 sets the linear CMIF could be obtained according to Alonso et al. (2010). Then, the nonlinear contribution to CMIF was straightforwardly calculated by subtracting it from the CMIF from the original signals.

Finally, both the linear and the nonlinear functions were characterized by the calculation of the area under their curve (CMIF vs $\tau$ ) (Alonso et al. 2010).

\subsection{Statistics and representation}

Spectral features and entropy were compared between the pre and post-stressor conditions by means of the nonparametric Wilcoxon signed-rank test with statistical significance set to 0.05 . Significance probability maps (SPM) indicating statistical differences due to the stress tests were obtained for the spectral and entropy variables. Stroop-induced effects were assessed by comparing the recording Re2 with the baseline (Re1) and sleep deprivation changes were calculated by comparing the recording $\mathrm{Re} 3$ to the baseline (see figure 1). 
Changes on EEG connectivity were represented by connectivity maps in which each significant difference is represented with a line connecting the two involved electrodes. To consider the effect of multiple comparisons, a threshold based on the binomial theorem was used, so that a single head can be considered significant only if the number of significant differences obtained exceeds the threshold. In other words, a minimum number of significant results was required before rejecting the overall hypothesis (Cross and Chaffin 1984). In this work, each pair of electrodes was compared using the Wilcoxon signed-rank test with significance set to 0.1 , whereas the overall significance for a connectivity map (whole head, 171 pairs) was set to 0.05 . Given these values, a minimum of 24 significant differences was established for each change direction: increase or decrease.

In addition to connectivity analysis via the MSC and CMIF maps, the average values of every variable at three regions of interest (anterior, interhemispheric, and global; all possible pairs of sensors inside each region considered) were compared between the pre and post-stressor conditions using the signed-rank test.

\section{Results}

\subsection{Univariate analysis}

Figure 2 shows the SPMs comparing changes of some selected spectral and entropy EEG variables calculated before and after the stress tests. The stressors assessed in this study correspond to different physiological processes that display various outcomes on the brain but share the effects related to stress. Absolute and relative powers showed the same trends (increases or decreases) in the changes. Theta activity is considered a marker of sleep propensity which enhances during the progression of wakefulness, and therefore, an increase in the absolute and relative theta powers was observed after the sleep deprivation but not after the Stroop test. Likewise, the alphal activity correlates with the alertness state, which in this study was reduced after the sleep deprivation but was enhanced after the Stroop test. This increase in the alphal activity indicated that the Stroop test stimulated the volunteers to a certain degree. A decrease in the high alpha band and an increase in the high beta band were detected, following the characteristic stress response on EEG spectra. On the other hand, both stressors showed significant decreases in approximate entropy values, located mainly at anterior sites.

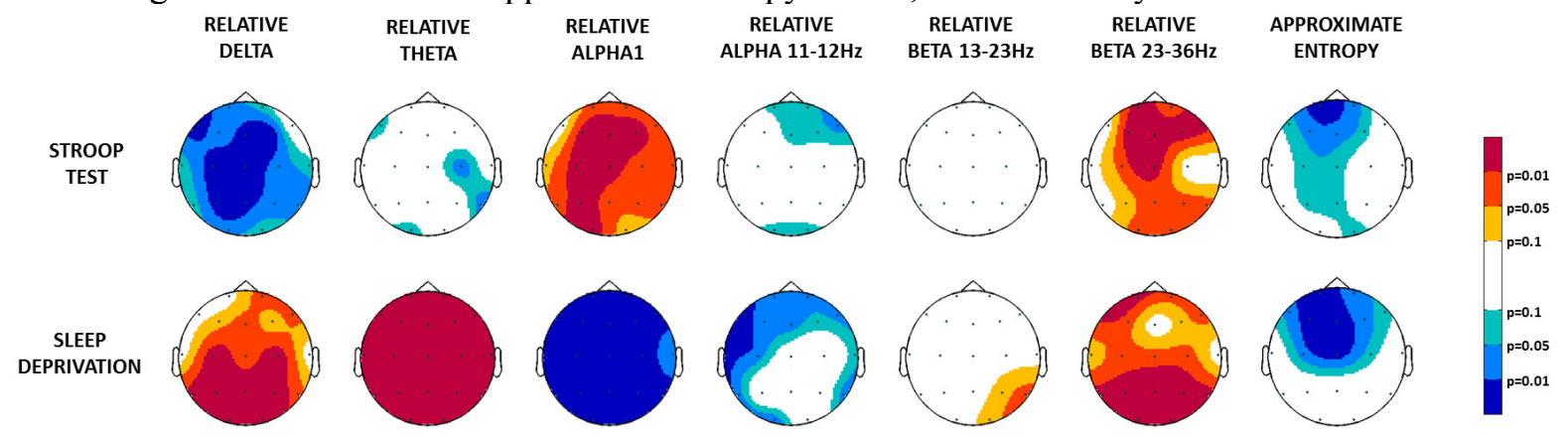

Figure 2. Significance probability maps showing differences after applying the stressor procedure (Stroop test or sleep deprivation) compared with a baseline situation, in the relative powers of delta, theta, alpha1, high alpha $(11-12 \mathrm{~Hz})$, low beta $(13-23 \mathrm{~Hz})$ and high beta $(23-36 \mathrm{~Hz})$, and in approximate entropy. Electrode positions are indicated by black dots. The 7-colour scale represents statistical differences based on p-values. Increases and decreases are depicted by hot and cold colours, respectively.

The results corresponding to the asymmetry index based on the EEG alpha power are listed in table 1. Although increases in the average asymmetry values after the stress tests suggest an activation shift from the left to the right hemisphere, no significant changes were obtained, probably due to intersubject variability (for overall frontal: $\mathrm{p}=0.125$ for sleep deprivation vs. baseline; and $\mathrm{p}=0.405$ for Stroop vs. baseline).

Table 1. Frontal EEG asymmetry index obtained for different conditions.

\begin{tabular}{cccc}
\hline ASI & Baseline & Stroop & Sleep deprivation \\
\hline
\end{tabular}




\begin{tabular}{cccc}
\hline RIGHT $_{\text {FRONTAL-LEFT }}$ FRONTAL & $-0.012 \pm 0.112$ & $0.002 \pm 0.124$ & $0.043 \pm 0.137$ \\
Fp2-Fp1 & $-0.010 \pm 0.072$ & $0.010 \pm 0.085$ & $0.024 \pm 0.118$ \\
F4-F3 & $0.016 \pm 0.112$ & $0.030 \pm 0.124$ & $0.045 \pm 0.103$ \\
F8-F7 & $-0.056 \pm 0.222$ & $-0.059 \pm 0.218$ & $0.047 \pm 0.333$ \\
\hline
\end{tabular}

\subsection{EEG connectivity}

The connectivity maps corresponding to the MSC variables related to the same bands in figure 2 are shown in figure 3 (not significant maps are depicted empty). Apart from the theta coherence, which presented a significant map for the Stroop test and a non-significant one for the sleep deprivation, all the MSC variables related to the sleep deprivation showed significant connectivity maps, but the direction of the observed changes depended on the spectral band and the location on the scalp. Coherence in the alpha bands showed decreases in the anterior region of the scalp, whereas this behaviour was not reproduced for the Stroop test case.

On the other hand, high beta coherence revealed significant increases of coherence spread all over the scalp for the deprivation case, and similar effects of the Stroop test located in the regions close to the sagittal middle line of the head.

The connectivity maps corresponding to the area under the CMIF for the sleep deprivation are depicted in figure 4. The Stroop test did not produce a significant map, neither for the linear nor for the nonlinear contributions of the CMIF. The sleep deprivation induced a significant decrease of the area for the linear case, spread all over the scalp. On the other hand, the nonlinear area showed increases mainly located at the anterior, central and temporal regions, in a clear contrast to the spreading of the linear case.

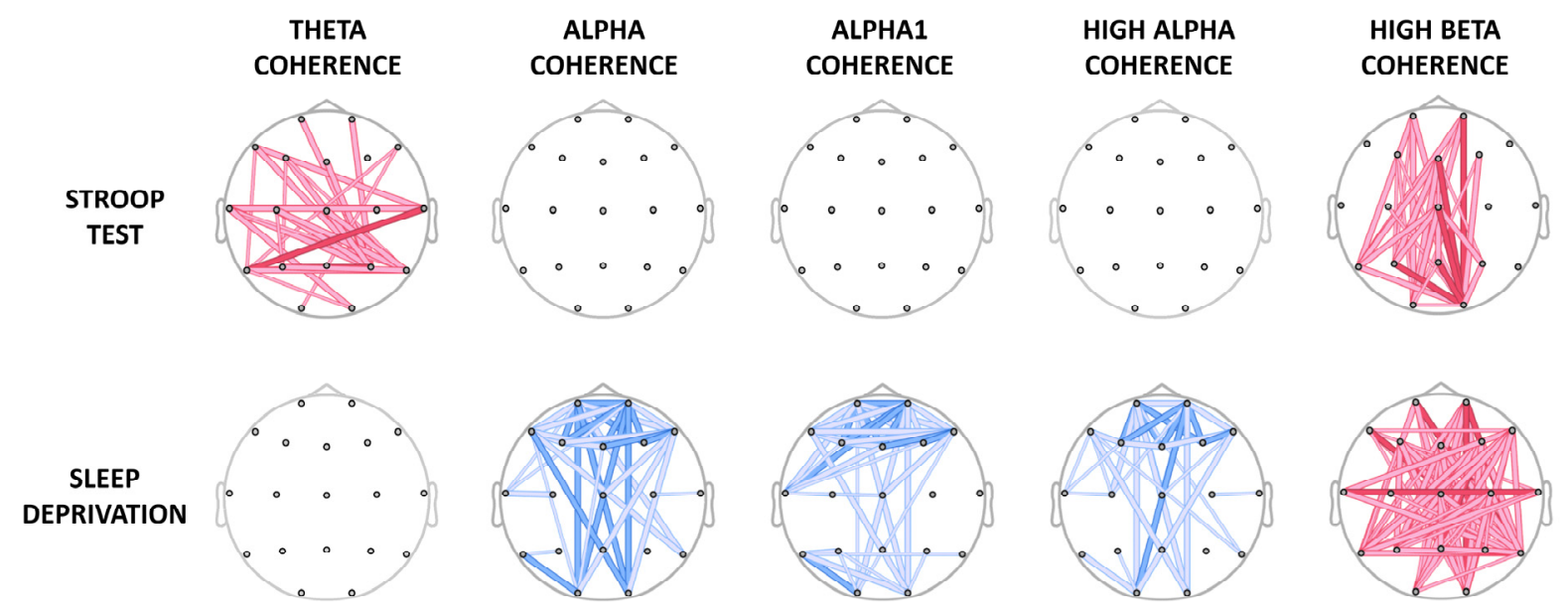

Figure 3. Connectivity maps for the selected coherence variables (theta, alpha, alpha1, alpha11-12Hz and beta $23-36 \mathrm{~Hz}$ ). Lines indicate significant changes $(\mathrm{p}<0.1)$ in each variable between the connected electrodes induced by the stressors: the Stroop test (upper row) and the sleep deprivation test (lower row). Warm and cold colours indicate significant increases and decreases, respectively, whereas line width and colour intensity denote the associated probability values: $\mathrm{p}<0.1$ for light and thin, $\mathrm{p}<0.05$ for light and thick; and $\mathrm{p}<0.01$ for dark and thick lines. Only maps whose total number of lines exceeds the overall significance threshold are depicted, the others are shown empty. 


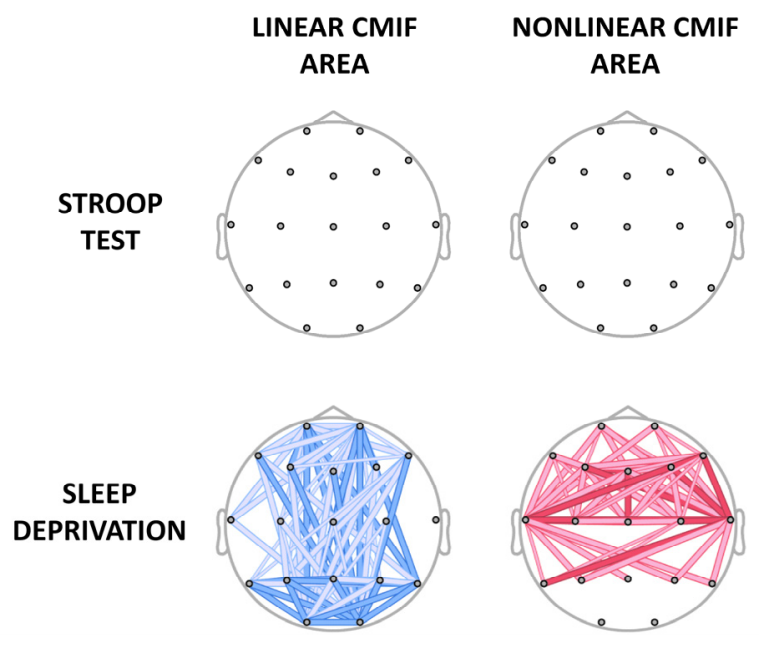

Figure 4. Connectivity maps for the area variable obtained from linear (left column) and nonlinear (right column) contributions of CMIF. For more details on line meaning, see legend of figure 3.

\subsection{Immunology assessment and average EEG variables}

Finally, table 2 shows a summary of the stress response obtained from the immunology assessment and the EEG analysis. The experimental data regarding the immune system, only available for the sleep deprivation experiment, showed that levels of IgA increased significantly after the test. The number of natural killer (NK) and T cells increased after 30h of sleep deprivation, but B-lymphocytes did not show significant differences.

The performance of the average values of the classical spectral variables (anterior region and whole-scalp) was also evaluated and their probability values and change direction are also shown in table 2. Both the stressors caused a decrease of the absolute power in the high alpha band and an increase of absolute power in the high beta band, located especially at anterior sites. In addition, the approximate entropy values decreased for both stressors. As shown by the probability values, this increase of EEG signal regularity was located mainly at the anterior regions of the scalp.

Table 2 includes the probability values and the direction of change for each MSC variable under the two stressors. After the sleep deprivation, all the averaged alpha coherence variables (corresponding to the alpha, alpha1, and high alpha bands) showed significant decreases at the anterior region, whereas alphal also evidenced significant decreases when interhemispheric and all possible pairs of channels were considered. On the contrary, the average high beta coherence did not display significant increases at the anterior zone, but it did when interhemispheric pairs or the whole-head were considered. None of these averaged variables showed significant results for the Stroop test.

As for the mutual information evaluation, average values for variables extracted from linear and nonlinear CMIF are included in table 2. The area under the CMIF showed significant results for the sleep deprivation stressor in the three considered regions, both for the linear and the nonlinear contributions. Linear area decreased whereas nonlinear increased, in agreement with the maps shown in figure 4. In the Stroop test case only the nonlinear interhemispheric evaluation evidenced a significant increase between pre and post-stressor recordings. 
Table 2. Summary of stress response obtained from the immune system and the EEG signals.

SLEEP DEPRIVATION

\begin{tabular}{|c|c|c|c|c|}
\hline Variable & $\begin{array}{l}\text { Direction of change } \\
\text { (increase/decrease) }\end{array}$ & p-value & $\begin{array}{l}\text { Direction of change } \\
\text { (increase/decrease) }\end{array}$ & p-value \\
\hline \multicolumn{5}{|l|}{ IMMUNE SYSTEM } \\
\hline $\operatorname{Ig} \mathrm{A}$ & $\uparrow$ & $<10^{-4}$ & & n. a. \\
\hline Lymphocytes B & $\uparrow$ & 0.484 & & n. a. \\
\hline Lymphocytes NK & $\uparrow$ & 0.004 & & n. a. \\
\hline Lymphocytes T (CD3) & $\uparrow$ & 0.041 & & n. a. \\
\hline \multicolumn{5}{|l|}{ UNIVARIATE ANALYSIS } \\
\hline$<$ RELPOW $\alpha_{11-12 \mathrm{~Hz}}>$ anterior & $\downarrow$ & 0.013 & $\downarrow$ & 0.059 \\
\hline$<$ RELPOW $\alpha_{11-12 \mathrm{~Hz}}>$ global & $\downarrow$ & 0.015 & $\downarrow$ & 0.139 \\
\hline$<$ RELPOW $\beta_{23-36 \mathrm{~Hz}}>$ anterior & $\uparrow$ & 0.013 & $\uparrow$ & 0.004 \\
\hline$<$ RELPOW $\beta_{23-36 \mathrm{~Hz}}>_{\text {global }}$ & $\uparrow$ & 0.004 & $\uparrow$ & 0.072 \\
\hline$<\mathrm{APEN}$ m=3;rOPT $>_{\text {anterior }}$ & $\downarrow$ & 0.012 & $\downarrow$ & 0.041 \\
\hline$<$ APEN $_{\mathrm{m}=3 ; \mathrm{rOPT}}>_{\text {global }}$ & $\downarrow$ & 0.120 & $\downarrow$ & 0.111 \\
\hline ASI frontal (Right-Left) & $\uparrow$ & 0.125 & $\uparrow$ & 0.405 \\
\hline \multicolumn{5}{|l|}{ CONNECTIVITY VARIABLES } \\
\hline$<\operatorname{MSC} \alpha>_{\text {anterior }}$ & $\downarrow$ & 0.002 & & n.s. \\
\hline$<\operatorname{MSC} \alpha>_{\text {global }}$ & & n.s. & & n.s. \\
\hline$<\operatorname{MSC} \alpha>$ interhemispheric & & n.s. & & n.s. \\
\hline$<\operatorname{MSC} \alpha_{1}>$ anterior & $\downarrow$ & $<0.001$ & & n.s. \\
\hline$<\operatorname{MSC} \alpha_{1}>_{\text {global }}$ & $\downarrow$ & 0.048 & & n.s. \\
\hline$<\operatorname{MSC} \alpha_{1}>$ interhemispheric & $\downarrow$ & 0.027 & & n.s. \\
\hline$<\mathrm{MSC} \alpha_{11-12 \mathrm{~Hz}}>$ anterior & $\downarrow$ & 0.012 & & n.s. \\
\hline$<\mathrm{MSC} \alpha_{11-12 \mathrm{~Hz}}>_{\text {global }}$ & & n.s. & & n.s. \\
\hline$<\mathrm{MSC} \alpha_{11-12 \mathrm{~Hz}}>_{\text {interhemispheric }}$ & & n.s. & & n.s. \\
\hline$<\mathrm{MSC} \beta_{23-36 \mathrm{~Hz}}>$ anterior & & n.s. & & n.s. \\
\hline$<$ MSC $\beta_{23-36 \mathrm{~Hz}}>_{\text {global }}$ & $\uparrow$ & 0.012 & & n.s. \\
\hline$<\mathrm{MSC} \beta_{23-36 \mathrm{~Hz}}>$ interhemispheric & $\uparrow$ & 0.011 & & n.s. \\
\hline$<$ Linear CMIF area $>$ anterior & $\downarrow$ & 0.007 & & n.s. \\
\hline$<$ Linear CMIF area $>_{\text {global }}$ & $\downarrow$ & 0.015 & & n.s. \\
\hline$<$ Linear CMIF area $>$ interhemispheric & $\downarrow$ & 0.008 & & n.s. \\
\hline$<$ Nonlinear CMIF area $>_{\text {anterior }}$ & $\uparrow$ & 0.007 & $\uparrow$ & 0.096 \\
\hline$<$ Nonlinear CMIF area $>_{\text {global }}$ & $\uparrow$ & 0.038 & & n.s. \\
\hline$<$ Nonlinear CMIF area $>_{\text {interhemispheric }}$ & $\uparrow$ & 0.002 & $\uparrow$ & 0.032 \\
\hline
\end{tabular}

n.s.: not significant; n.a.: not available.

\section{Discussion and conclusions}

In this study, the changes induced in the brain by two different stressors, namely Stroop test (psychological stressor) and sleep deprivation (physical stressor), were assessed in order to identify common EEG patterns that could characterize stressing conditions. The stress-induced effects on spontaneous EEG signals were evidenced by means of classic spectral measures, the asymmetry index, approximate entropy, and connectivity variables obtained from coherence and cross mutual information function. Initially, all these variables were calculated and their performances were compared between the pre- and post-stressor conditions by means of Wilcoxon signed rank tests. Subsequently, their mean values at different regions (anterior and interhemispheric, given their 
importance according to the literature, and whole-head, to have a general measure of the effects) were also obtained and evaluated using the same statistical test.

The classic spectral variables high alpha (11 to $12 \mathrm{~Hz})$ and high beta $(23$ to $36 \mathrm{~Hz})$ showed the representative stress-related decrease and increase for both stressors, respectively, in agreement with the study of Thompson and Thompson (2007). Sleep deprivation can be considered a broad situation that affects cognitive function and involves different effects related to fatigue, clumsiness, daytime sleepiness, and stress. Consequently, the theta activity was clearly increased for the deprivation case but no significant changes were obtained for the Stroop test, because theta power $(3.5$ to $7.5 \mathrm{~Hz})$ is closely related to homeostatic sleep pressure (Finelli et al. 2000). Likewise, the alpha1 activity (7.5 to $10.5 \mathrm{~Hz}$ ) indicated an increase of attention and alertness for the Stroop test and a decrease for the sleep deprivation test related to fatigue. Interestingly, a noticeable significant decrease in the high alpha activity (11 to $12 \mathrm{~Hz}$ ) was found in the anterior region of the scalp for both experimental stressor tests. These results are in accordance with the studies that highlight this area as very important to understand the asymmetry of the alpha EEG activity under different conditions such as stress, depression and sleep deprivation (Ferreira et al. 2006, Hinrikus et al. 2009, Seo and Lee 2010). However, the obtained values of the alpha EEG asymmetry index did not show significant changes, although their average values suggested an activation shift from the left to the right hemisphere (see table I). It is clear that this metric suffers from some limitations and it should be replaced by specific hemispheric analyses in order to understand the precise nature of cortical asymmetries and relationships (Coan and Allen 2004).

The corresponding probability values of the selected averaged spectral variables are presented in table 2, and reinforce the importance of the thorough study of the activity on the frontal area of the brain to reveal stress-related effects, as the results were significant for both stressors and showed a decrease and an increase of high alpha and high beta powers, respectively.

The approximate entropy values also exhibited a consistent behaviour, displaying a decreasing anterior pattern after both stressors (see figure 2). These results were confirmed by the significant decrease obtained in the anterior zone of the scalp (see table 2) in accordance with the results obtained of Tran et al. with subjects during a driving simulation task (Tran et al. 2007). Decreased entropy values, due to both stressors under study, may be associated to deficient information processing (Abásolo et al. 2008).

To summarize univariate analysis, the high alpha and high beta powers and the entropy variables showed statistically significant changes associated with both a psychological and a physical stressor. In addition, the much lower probability values obtained for the sleep deprivation with respect to the Stroop test could be an indicator of the lightness of the latter.

Regarding the immune system, although several studies have assessed the effect of sleep deprivation on the immune cell number, the results have been inconsistent, which might be explained in part by different durations of the sleep deprivation experiments. For example, it has been reported a decrease NK cell count after 40 hours, but an increased count after 64 hours of sleep deprivation (Dinges et al. 1994). In our study the lymphocytes count increased (especially T and NK cells), and so did immunoglobulin A, indicating the activation of the immune system as a result of an acute stress situation.

Regarding the results of the EEG connectivity measures, the magnitude squared coherence (MSC) revealed linear connectivity changes associated with each spectral band. Significant global maps, according to the Cross-Chaffin binomial test, were obtained in the theta and high beta bands for the Stroop test, whereas only in the latter band for the sleep deprivation. These high beta increases were spread all over the scalp for the deprivation and located around the sagittal middle line of the head for the Stroop test. Such increases indicate a boost of cortical connectivity in this band, considered a busy brain indicator (Thompson and Thompson 2007) in univariate analysis. They also suggest higher synchronization associated with mental load, increased difficulty perception and lack of ability to relax quickly, even after only $10 \mathrm{~min}$ of Stroop test, that univariate variables characterize as a light stressor as explained above. Interestingly, the coherence under stress also shows some gradation between both tests. Note that not only the increase in the absolute high beta power but also its linear relationship among electrodes, as quantified by the coherence, suggests this spectral band as a potential stress marker. On the other hand, the alpha coherence variables (alpha, alpha1, and high 
alpha) displayed maps with significant decreases only for the sleep deprivation case, especially in the anterior region.

Significant decreases in the high alpha power were obtained for both type of stressors, especially for the deprivation test, but a decrease in coherence was only found in this test. It seems that the effects of stress on EEG connectivity are more difficult to produce or hard to detect than the cerebral activity measured by univariate analyses. This might be the reason why only the high beta coherence changes significantly under the Stroop test, whereas all the alpha coherence variables presented significant changes for the sleep deprivation.

Concerning the results obtained for the area under the CMIF, the Stroop test did not show any significant map. On the contrary, sleep deprivation was associated to widespread significant decreases of the linear area, whereas the nonlinear area evidenced significant increases located mainly over the anterior, central, and temporo-parietal regions of the head. These opposite trends have already been obtained in previous connectivity studies evaluating drug effects and have been associated with different phenomena which in turn could be related to different cortico-cortical synchronization changes, and also to changes in the relationship between cortical and subcortical structures (Alonso et al. 2010). In this work, these trends might be associated with the increased alertness and attention and with the increased mental load caused by a stressor, but with only the information of the statistical maps no further inference can be concluded. With respect to the average values of the CMIF, variables displayed significant statistical differences in all the regions for the sleep deprivation case, but only changes in the nonlinear CMIF area were found for the Stroop test: a trend for the anterior region $(\mathrm{p}<$ $0.1)$ and a significant increase $(p=0.036)$ in the interhemispheric variable. Hence, although it is clear that the changes in connectivity triggered by this stressor were light and not detectable by means of linear connectivity measures, the nonlinear variable was able to reveal an increase in the coupling between the two hemispheres. This coupling detected by CMIF exhibited many significant changes due to the sleep deprivation but the Stroop test seemed too light to produce similar effects on EEG connectivity.

It is important to note that the obtained decreases of alpha coherence and linear CMIF area and the increases of beta coherence and nonlinear CMIF area offer different point of views of the phenomenon under study. On the one hand, taking into account the magnitude of the observed changes, both global coherence and CMIF decreased because alpha power dominated over beta power. On the other hand, similar trends between alpha power (or coherence) and linear CMIF, and between beta power (or coherence) and nonlinear CMIF, have also been observed in previous works when studying psychopharmacological effects (Alonso et al. 2010, 2012). They concluded that these results may indicate decreased connectivity between cortical and subcortical structures and increased cognitive efficiency (Adler and Brasen 2001), but all the obtained results should be interpreted in a statistical sense because no direct physiological connections were studied, and other possible mechanisms could explain the observed changes.

The frontal asymmetry index did not show statistically significant changes produced by any of the stressors. The limitations of this metric have been mentioned above, and an alternative index associated with the relationship between the two hemispheres might be more useful, like the one proposed in this work that showed changes under both tests and especially after the sleep deprivation. The interhemispheric nonlinear couplings measured by the CMIF increased significantly because of stress even after the Stroop test.

Results of the study suggest that some reliable stress indexes may be obtained from EEG signals. High beta coherence showed significant differences in maps corresponding to both stressors, and the nonlinear interhemispheric area under the CMIF also revealed itself as a valid stress-related measure, showing differences after both stress tests. Sleep deprivation induces different effects related to behavioural, physical, metabolic, and cognitive processes, some of them associated with stress. Regarding the Stroop test, only mental load is induced and depending of the duration of experimental test this load can be light. Nevertheless, some common patterns between the two stressors were found, because both promoted decreases in frontal relative high alpha power, as expected, but also in frontal approximate entropy, as well as increases in beta power, beta coherence and nonlinear connectivity as measured by CMIF. Changes of these measures were more intense or more extended over the scalp during sleep deprivation. Thus, these findings suggest more increased alertness and higher mental load with respect to the Stroop test, which both effects could well be stress-related, although further 
investigation should be performed to validate these measures as useful mental stress markers. However, these EEG-based stress measures could be a useful and non-invasive tool to assess and control stress and its effects on the course of disorders, such as cardiovascular and neurological diseases. Future work on these indexes could lead to the calculation and assessment of a combined EEG-based stress index based on spectral variables (high alpha and high beta) and connectivity measures (coherence and mutual information function) related to linear and nonlinear couplings.

\section{Acknowledgements}

This study was supported by the Ministerio de Ciencia e Innovación, under contract DPI 2011-22680. CIBER-BBN is an initiative of the Instituto de Salud Carlos III, Spain. The authors would also like to thank: the staff at the Centre d'Investigació de Medicaments de l'Institut de Recerca de l'Hospital de la Santa Creu i Sant Pau for their technical assistance; Dr. M. Portella and E. Grasa from CIBERSAM for their assistance with the Stroop task and the psychological tests; and BIOIBERICA S.A. for their support.

\section{References}

Abásolo D, Escudero J, Hornero R, Gómez C and Espino P 2008 Approximate entropy and auto mutual information analysis of the electroencephalogram in Alzheimer's disease patients Medical \& Biological Engineering \& Computing 46 1019-1028

Adler G, Brassen S 2001 Short-term rivastigmine treatment reduces EEG slow-wave power in Alzheimer patients. Neuropsychobiology 43 273-276.

Ackermann K, Revell V L, Lao O, Rombouts E J, Skene D J and Kayser M 2012 Diurnal rhythms in blood cell populations and the effect of acute sleep deprivation in healthy young men Sleep 35 933-940

Alonso J F, Mañanas M A, Romero S, Hoyer D, Riba J and Barbanoj M J 2010 Drug effect on EEG connectivity assessed by linear and nonlinear couplings Human Brain Mapping 31 487-497

Alonso J F, Mañanas M A, Romer, S, Rojas-Martínez M and Riba J 2012 Cross-conditional entropy and coherence analysis of pharmaco-EEG changes induced by alprazolam Psychopharmacology $221397-406$

Bollinger T, Bollinger A, Skrum L, Dimitrov S, Lange T and Solbach W 2009 Sleep-dependent activity of T cells and regulatory T cells, Clinical \& Experimental Immunology, 155 231-238

Bruhn J, Röpcke H, Rehberg B, Bouillon T and Hoeft A 2000 Electroencephalogram approximate entropy correctly classifies the occurrence of burst suppression pattern as increasing anesthetic drug effect Anesthesiology 93 981-985

Coan J A and Allen J J B 2004 Frontal EEG asymmetry as a moderator and mediator of emotion Biological Psychology, 67 7-49

Cross E M and Chaffin W W 1982 Use of the binomial theorem in interpreting results of multiple tests of significance Educational and Psychological Measurement 42 25-34

Davidson R J 1993 Cerebral asymmetry and emotion conceptual and methodological conundrums Cognition and Emotion 7 115-138

Davidson, R J 2004 What does the prefrontal cortex "do" in affect: Perspectives on frontal EEG asymmetry research Biological Psychology 67 219-233.

De Gennaro L, Vecchio F, Ferrara M, Curcio G, Rossini P M and Babiloni C 2005 Antero-posterior functional coupling at sleep onset changes as a function of increased sleep pressure Brain Research Bulletin 65 133-140

Dinges D F, Douglas S D, Zaugg L, Campbell D E, McMann J M, Whitehouse W G, Orne E, Kapoor S C, Icaza E and Orne M T 1994 Leukocytosis and natural killer cell function parallel neurobehavioral fatigue induced by 64 hours of sleep deprivation The Journal of Clinical Investigation, 93 1930-1939

Ferreira C, Deslandes A, Moraes H, Cagy M, Basile LF, Piedade R and Ribeiro P 2006 The relation between EEG prefrontal asymmetry and subjective feelings of mood following 24 hours of sleep deprivation Arquivos de Neuro-Psiquiatria 64 382-387

Finelli L A, Baumann H, Borbély A A and Achermann P 2000 Dual electroencephalogram markers of human sleep homeostasis correlation between theta activity in waking and slow-wave activity in sleep Neuroscience 101 523-529 
Hamer M, Boutcher YN and Boutcher SH 2003 The role of cardiopulmonary baroreceptors during the forearm vasodilatation response to mental stress in humans Psychophysiology 40 249-253

Hinrikus H, Suhhova A, Bachmann M, Aadamsoo K, Vohma U, Lass J and Tuulik V 2009 Electroencephalographic spectral asymmetry index for detection of depression Medical \& Biological Engineering \& Computing 47 1291-1299

Irwin M, Mcclintick J, Costlow C, Fortner M, White J and Gillin C 1996 Partial night sleep deprivation reduces natural killer and cellular immune responses in humans $\boldsymbol{F A S E B}$ Journal 10 643-653

Kar S, Routray A and Nayak B P 2011 Functional network changes associated with sleep deprivation and fatigue during simulated driving Validation using blood biomarkers Clinical Neurophysiology 122 966-974

Koenis M M G, Romeijn N, Piantoni G, Verweij I, Van der Werf Y D, Van Someren E J W and Stam CJ 2011 Does sleep restore the topology of functional brain networks? Human Brain Mapping 34 487-500

Lederbogen F, Kirsch P, Haddad L, Streit F, Tost H, Schuch P, Wüst S, Pruessner J C, Rietschel M, Deuschle M and Meyer-Lindenberg A 2011 City living and urban upbringing affect neural social stress processing in humans Nature 474 498-501

Lewis RS, Weekes NY and Wang TH 2007 The effect of a naturalistic stressor on frontal EEG asymmetry, stress and health Biological Psychology 75 239-247

Lu S, Chen X, Kanters J K, Solomon I C and Chon K H 2008 Automatic selection of the threshold value R for approximate entropy IEEE Transactions on Biomedical Engineering 55 1966-1972

Manuck S B, Cohen S, Rabin B S, Muldoon M F and Bachen E A 1991 Individual differences in cellular immune response to stress Psychological Science 2 111-115

McEwen B S 2006 Sleep deprivation as neurobiologic and physiologic stressor allostasis and allostatic load Metabolism Clinical and Experimental 55 20-23

$\mathrm{Na}$ S H, Jin S H and Kim S Y 2006 The effects of total sleep deprivation on brain functional organization Mutual information analysis of waking human EEG International Journal of Psychophysiology 62 238-242

Papadelis C, Chen Z, Kourtidou-Papadeli C, Bamidis P D, Chouvarda I, Bekiaris E and Maglaverasa N 2007 Monitoring sleepiness with on-board electrophysiological recordings for preventing sleep-deprived traffic accidents Clinical Neurophysiology 118 1906-1922

Pruett S B 2003 Stress and the immune system Pathophysiology 9 133-153

Seo S H and Lee J T 2010 Stress and EEG Convergence and hybrid information technologies ed M Crisan (Croatia: Intech) pp 413-326

Schreiber T and Schmitz A 2000 Surrogate time series Physica D 142 346-382

Stroop J R 1935 Studies of interference in serial verbal reactions Journal of Experimental Psychology 18 643-662

Sulaiman N, Taib M N, Lias S, Murat Z H, Aris S A M, Mustafa M and Rashid N A (2012) Development of EEG-based stress index Proceedings of the International Conference on Biomedical Engineering (Penang) pp 461-466

Taheri S, Lin L, Austin D, Young T and Mignot E 2004 Short sleep duration is associated with reduced leptin, elevated ghrelin, and increased body mass index PLoS Medicine 1 210-217

Thompson M and Thompson L 2007 Neurofeedback for stress management Principles and practice of stress management ed P M Lehrer, R L Woolfolk, W E Sime (New York: The Guilford Press) pp 249-290.

Tran Y, Thuraisingham R A, Wijesuriya N, Nguyen H T and Craig A 2007 Detecting neural changes during stress and fatigue effectively a comparison of spectral analysis and sample entropy Proceedings of the 3rd International IEEE/EMBS Conference on Neural Engineering (Kohala Coast, HI), pp 350-353

Tulen J H, Moleman P, van Steenis H G and Boomsma F 1989 Characterization of stress reaction to the Stroop Color Word Test Pharmacology Biochemistry and Behavior 32 9-15

Woolfolk R L, Lehrer P M and Allen L A 2007 Conceptual issues underlying stress management Principles and practice of stress management ed P M Lehrer, R L Woolfolk, W E Sime (New York: The Guilford Press) pp 3-16 